\title{
GC7 enhances cisplatin sensitivity via STAT3 signaling pathway inhibition and eIF5A2 inactivation in mesenchymal phenotype oral cancer cells
}

\author{
LIANG FANG, LI GAO, LEI XIE and GUIZHOU XIAO
}

Department of Head and Neck Surgery, Sir Run Run Shaw Hospital, School of Medicine, Zhejiang University, Hangzhou, Zhejiang 310016, P.R. China

Received May 19, 2017; Accepted November 24, 2017

DOI: 10.3892/or.2017.6161

\begin{abstract}
Eukaryotic initiation factor 5A2 (eIF5A2), a newly identified oncogene, promotes cell survival, proliferation and motility in tumorigenesis. Drug resistance and dose-related adverse side-effects greatly reduce the efficiency and safety of cisplatin-based chemotherapy in advanced or recurrent oral squamous cell carcinoma (OSCC) patients. The present study investigated the effect of eIF5A2 combined with N1-guanyl1,7-diaminoheptane (GC7, a novel eIF5A2 inhibitor) or siRNA. We found that low concentrations of GC7 $(\leq 5 \mu \mathrm{M})$ had little effect on OSCC cell viability, but significantly enhanced cisplatin cytotoxicity. Compared with cisplatin, GC7/cisplatin had little effect on cisplatin-promoted mesenchymal-epithelial transition in mesenchymal phenotype Tca8113 and HN30 cells, or on cisplatin-induced epithelial-mesenchymal transition (EMT) in epithelial phenotype Cal27 and HN4 cells. Further research revealed that the upregulation of p-STAT3 and c-Myc which was induced by the single treatment with either cisplatin or GC7 was significantly reversed by the GC7/cisplatin combination in mesenchymal phenotype Tca8113 and HN30 cells. In in vivo treatment, we revealed that the GC7/cisplatin combination presented significant tumor volume reduction without distinct body weight loss. In conclusion, our data indicated that eIF5A2 is a potent therapeutic target in OSCC treatment. Our results revealed a novel mechanism by which GC7/cisplatin combination therapy may offer an efficient and safe therapeutic alternative to advanced or recurrent OSCC patients.
\end{abstract}

\section{Introduction}

In 2015, there were 45,780 new cases of cancer of the oral cavity and pharynx diagnosed with $90 \%$ of them attributed

Correspondence to: Dr Liang Fang, Department of Head and Neck Surgery, Sir Run Run Shaw Hospital, School of Medicine, Zhejiang University, 3 East Qingchun Road, Hangzhou, Zhejiang 310016, P.R. China

E-mail: fangliang_dr@zju.edu.cn

Key words: eIF5A2, N1-guanyl-1,7-diaminoheptane, GC7, OSCC, EMT, STAT3, cisplatin to oral squamous cell carcinoma (OSCC) and 8,650 people succumbed to this disease, since $69.4 \%$ of these new cases were diagnosed at late stage III and IV of the disease (1). Therapeutic treatment of OSCC is based on the stage of the disease and includes, either separately or in combination, surgery, radiation and chemotherapeutic agents such as cisplatin (2-4). However, despite many novel chemotherapeutic agents and molecularly targeted therapies, the essential molecular mechanisms underlying the pathogenesis of OSCC and chemotherapy resistance have yet to be fully elucidated, which is important especially to late-stage patients, since the 5-year survival rates have not significantly improved in decades $(5,6)$.

The initiation of oral cancer cells in primary tumor invasion-metastasis cascade is enabled by epithelial-mesenchymal transition (EMT), a complex and reversible process which leads to loss of epithelial markers, such as E-cadherin and gain of mesenchymal markers, such as $\mathrm{N}$-cadherin, fibronectin and vimentin with increased migration and invasive capabilities (7). EMT is initiated and regulated by various different cytokines and growth factors during tumor progression (8). The gain of EMT markers has been associated with the resistance of ovarian carcinoma epithelial cell lines to paclitaxel (9). Although emerging evidence indicates that OSCCs which undergo EMT are responsible for radio-chemoresistance, the underlying mechanism remains unclear (10-12). Therefore, considering the oncogenic potential of EMT, it is essential to investigate the possible role of EMT in drug resistance to commonly used OSCC chemotherapeutic agents such as cisplatin, in order to acquire promising improvements.

The presence of recurrent copy number aberrations of $3 q$ is one of the most frequent chromosomal aberrations in OSCC and is related to a more substantial risk of metastasis $(11,13)$. To date, eukaryotic translation initiation factor 5A-2 (eIF5A2) located on 3q26 is the only known substrate of deoxyhypusine synthase (DHPS), an enzyme catalyzing hypusination that contributes to the progression of tumor malignancy and poor prognosis $(14,15)$. The overexpression of eIF5A2 has been demonstrated to be related to cancer metastasis in hepatocellular and colorectal carcinomas and to poor prognosis in bladder and ovarian cancers (16-18). Several studies have found that N1-guanyl-1,7-diaminoheptane (GC7), a novel DHPS inhibitor, suppresses tumor cell proliferation by inhib- 
iting eIF5A2 (18-22). These findings indicated that aberrant eIF5A2 expression was a response to the malignant behavior of cancer cells. However, the potential oncogenic role and the underlying molecular mechanisms of eIF5A2 in OSCC have not been elucidated.

In addition, EMT progression was significantly impacted by eIF5A 2 through different molecular pathways in many solid tumors $(19,20,22,23)$. In the present study, we examined the antitumor effect of cisplatin-based treatment combined with GC7 in OSCC cells. We also investigated the possible underlying molecular mechanisms through which the activation of eIF5A2 was involved in the enhanced cisplatin sensitivity in OSCC cells and found that the inactivation of eIF5A2 induced by GC7 increased cisplatin chemosensitivity in mesenchymal phenotype OSCC cells via inhibition of the signal transducer and activator of transcription 3 (STAT3) signaling pathway feedback activation.

\section{Materials and methods}

Cell culture and reagents. The human OSCC cell lines, Cal27, HN4, HN30 and Tca8113, were obtained from the Chinese Oral Tissue Culture and Collection Center (Shanghai, China) and were maintained as monolayers in RPMI-1640 medium (Gibco, Carlsbad, CA, USA) with $10 \%$ fetal bovine serum (FBS; HyClone Laboratories, Logan, UT, USA), 1\% penicillin/streptomycin (Sigma-Aldrich, St. Louis, MO, USA) in $5 \% \mathrm{CO}_{2}$ at $37^{\circ} \mathrm{C}$. Cisplatin was obtained from Sigma-Aldrich and stock solutions were prepared in dimethyl sulphoxide (DMSO). GC7 was obtained from Calbiochem (Merck KGaA, Darmstadt, Germany). Unless otherwise indicated, all other chemicals were the purest grade available and were obtained from Sigma-Aldrich.

CCK-8 cell viability assay and EdU incorporation assay. Cell viability was analyzed with the Cell Counting Kit-8 (CCK-8; Dojindo Laboratories, Kumamoto, Japan) in accordance with the manufacturer's protocols. Briefly, OSCC cells $\left(3 \times 10^{3}\right.$ cells/well) were seeded onto 96-well culture plates, allowed to attach for $12 \mathrm{~h}$ and serum-starved overnight to synchronize the cells. Subsequently, the culture medium was replaced with complete medium containing cisplatin or cisplatin combined with GC7 at indicated doses for $48 \mathrm{~h}$. Subsequnelty, CCK-8 solution $(10 \mu \mathrm{l} /$ well) was added and the cells were incubated at $37^{\circ} \mathrm{C}$ for $3 \mathrm{~h}$, and then absorbance was assessed at $450 \mathrm{~nm}$ using an MRX II microplate reader (Dynex Technologies Inc., Chantilly, VA, USA). The cell viability was calculated as a percentage of untreated control cells. Assessement of the inhibition rate of cell proliferation was performed using a Click-iT EdU Imaging kit (Invitrogen Life Technologies, Carlsbad, CA, USA) following the manufacturer's protocol. Each experiment was performed in triplicate and repeated three times.

eIF5A2 siRNA transfection. OSCC cells were transfected with eIF5A2 siRNA or negative control siRNA (Santa Cruz Biotechnology, Inc., Dallas, TX, USA) using Lipofectamine 2000 (Invitrogen Life Technologies) according to the manufacturer's instructions. The transfection medium (Opti-MEM) was replaced with complete medium $6 \mathrm{~h}$ after transfection, and then the cells were incubated for $24 \mathrm{~h}$, before all subsequent experiments were performed. Each experiment was performed in triplicate and repeated three times.

Apoptosis analysis. For the evaluation of apoptosis in $\mathrm{HN} 30$ cells induced by cisplatin $\left(\mathrm{IC}_{30}\right.$ and $\mathrm{IC}_{50}$ ) alone or combined with $5 \mu \mathrm{M}$ GC7 for 48 and $24 \mathrm{~h}$, respectively, the Annexin V-FITC PI Apoptosis Detection kit (BD Pharmingen; BD Biosciences, San Diego, CA, USA) was used according to the manufacturer's protocols. The apoptosis cells were identified using an FC 500 flow cytometer (Beckman Coulter, Inc., Los Angeles, CA, USA) at $488 \mathrm{~nm}$. Each experiment was performed in triplicate and repeated three times.

Western blot analysis. OSCC cells were collected and whole cellular extracts were lysed in $50 \mu \mathrm{l}$ cell of lysis buffer (Cell Signaling Technology, Beverly, MA, USA) containing protease inhibitors (BioVision, Milpitas, CA, USA) according to the manufacturer's instructions. The protein concentration was quantified using the BCA Protein kit (Thermo Fisher Scientific, Rockford, IL, USA). Equal amounts of proteins were separated by $10 \%$ SDS-PAGE and transferred to PVDF membranes (Millipore, Billerica, MA, USA), blocked with TBS/T containing 5\% bovine serum albumin (BSA) for $2 \mathrm{~h}$ on ice, and then incubated with primary antibodies against E-cadherin (mouse monoclonal, ab1416), vimentin (mouse monoclonal, ab8978), eIF5A2 (mouse polyclonal, ab168122), p53 (mouse monoclonal, ab26), STAT3 (rabbit monoclonal, ab68153), p-STAT3 (rabbit monoclonal, ab76315), c-Myc (rabbit monoclonal, ab32072) or GAPDH (mouse monoclonal, ab8245) (1:1,000 diluted in TBS/T; Abcam, Cambridge, MA, USA) at $4^{\circ} \mathrm{C}$ overnight, washed three times with TBS/T and then incubated with the appropriate HRP-conjugated secondary antibodies (rabbit anti-mouse, ab6728; goat anti-rabbit, ab6721) (dilution 1:2,000; Abcam) for $1 \mathrm{~h}$ at room temperature. The protein bands were developed using chemiluminescence (GE Healthcare Life Sciences, Piscataway, NJ, USA) and visualized using autoradiography (Kodak, Rochester, NY, USA). The quantification of proteins was performed by estimation of the protein band densities using Image-Pro Plus 6.0 software (Media Cybernetics Inc., Bethesda, MD, USA) and the protein levels were standardized to GAPDH.

Establishment and treatment of tumor xenografts in vivo. All animal studies were carried out in compliance with the Guide for the Care and Use of Laboratory Animals of Zhejiang University (Zhejiang, China). All applicable international, national, and/or institutional guidelines for the care and use of animals were followed. Male athymic BALB/c nude mice (Shanghai Experiment Animal Centre, Shangai, China), 5-6 weeks old and weighing 15-20 g, were used in the present study. OSCC Tca8113 cells $\left(1 \times 10^{6}\right)$ suspended in $0.1 \mathrm{ml}$ phosphate buffered saline (PBS) were injected subcutaneously (s.c.) into the left flank of each mouse and tumor growth was monitored every other day. Tumor length (L) and width (W) were assessed with a sliding caliper and tumor volume was determined with the standard formula $(\mathrm{LxW} 2) /^{2}(24,25)$. Drug treatment was initiated when the tumor volume reached 30-75 $\mathrm{mm}^{3}$. Twenty nude mice injected with OSCC cells were randomly divided into four groups: three experimental groups including GC7 (1 mg/kg), cisplatin $(2 \mathrm{mg} / \mathrm{kg})$ and GC7 (1 mg/ 

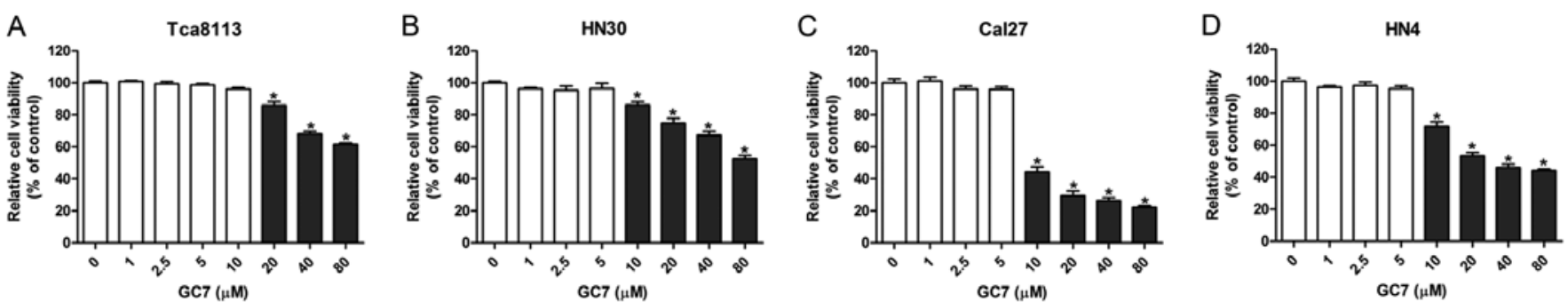

Figure 1. Low concentrations of GC7 have little effect on cytotoxicity against OSCC cells. (A) Tca8113, (B) HN30, (C) Cal27 and (D) HN4 cells were treated with different concentrations of GC7 for $48 \mathrm{~h}$. The CCK-8 values were normalized to the control group (control), which was treated without GC7 ("P<0.05), GC7, N1-guanyl-1,7-diaminoheptane; OSCC, oral squamous cell carcinoma.

$\mathrm{kg})+$ cisplatin $(2 \mathrm{mg} / \mathrm{kg})$, as well as one vehicle-treated control group (equal volume of diluents). The drugs were administered intraperitoneally (i.p.) every 2 days for 2 weeks. Subsequently, the mice were euthanized by cervical dislocation, and then the tumors were carefully dissected from each mouse for tumor weight assessement. The relative tumor inhibitory rate was calculated as follows: (mean tumor weight of the control group - mean tumor weight of the experimental group)/(mean tumor weight of the control group) $\mathrm{x} 100 \%$.

Statistical analysis. The results are presented as the mean \pm standard deviation (SD). Data were statistically analyzed using the SPSS 17.0 software (SPSS Inc., Chicago, IL, USA). The effects of the combined treatment were compared using two-way ANOVA, followed by Bonferroni's post hoc test. Analyses of the comparisons of two groups were carried out using Student's t-tests. $\mathrm{P}<0.05$ was considered to indicate a statistically significant difference.

\section{Results}

Low concentrations of GC7 exhibit slight cytotoxicity against OSCC cells. By inhibiting the hypusination of eIF5A2 by DHPS, GC7 specifically prevents the activation of eIF5A2. However, the cytotoxicity of GC7 against OSCC cells has rarely been reported. To determine the appropriate concentration of GC7 for co-administration with cisplatin, CCK-8 cell viability assays were performed in order to test the cytotoxicity of GC7 in Tca8113, Cal27, HN30 and HN4 OSCC cell lines. The results revealed that GC7 had almost no effect on Cal27, $\mathrm{HN} 30$ and $\mathrm{HN} 4$ cell viability at a concentration between 0 and $5 \mu \mathrm{M}$, however, GC7 significantly inhibited cell viability at a concentration exceeding $10 \mu \mathrm{M}$ (Fig. 1B-D). Similarly, the cell viability of Tca8113 cells was not affected when the GC7 concentration was $<10 \mu \mathrm{M}$, whereas it was significantly inhibited at a GC7 concentration exceeding $20 \mu \mathrm{M}$ (Fig. 1A). Our data indicated that low concentrations of GC7 exerted little cytotoxicity against OSCC cells. Several studies have reported that low concentrations of GC7 could inhibit the hypusination of eIF5A2 effectively in some tumor cells $(26,27)$. Subsequently, $5 \mu \mathrm{M} \mathrm{GC7}$, which exerted little cytotoxicity against OSCC cells but could inhibit the efficiency of eIF5A2 activation, was used for the following co-treatments with cisplatin.

GC7 enhances the sensitivity of cisplatin in OSCC cells through inhibition of cell proliferation without induction of
Table I. $\mathrm{IC}_{50}$ values of cisplatin in OSCC cell lines with or without $\mathrm{GC} 7$ treatment.

\begin{tabular}{lll}
\hline & \multicolumn{2}{c}{$\mathrm{IC}_{50}(\mu \mathrm{g} / \mathrm{ml})^{\mathrm{a}}$} \\
\cline { 2 - 3 } OSCC cell lines & \multicolumn{1}{c}{ Cisplatin } & \multicolumn{1}{c}{ Cisplatin + GC7 } \\
\hline Cal27 & $7.729(4.162-11.296)$ & $2.994(2.611-3.377)^{\mathrm{b}}$ \\
HN4 & $1.797(1.722-1.872)$ & $1.215(1.164-1.266)^{\mathrm{b}}$ \\
HN30 & $2.201(1.971-2.431)$ & $1.000(0.955-1.046)^{\mathrm{b}}$ \\
Tca8113 & $1.685(1.535-1.835)$ & $0.818(0.787-0.850)^{\mathrm{b}}$ \\
\hline
\end{tabular}

${ }^{\mathrm{a}} \mathrm{IC}_{50}$ values and $95 \%$ confidence interval of cisplatin in each treatment. ${ }^{\mathrm{b}} \mathrm{P}<0.05$ vs. cisplatin alone. OSCC, oral squamous cell carcinoma; GC7, N1-guanyl-1,7-diaminoheptane.

apoptosis. To assess the synergistic cytotoxic effect of cisplatin plus GC7, we used a CCK-8 assay to assess cell viability and EdU incorporation assay to test the inhibition of proliferation of OSCC cells treated with cisplatin alone or cisplatin plus GC7 for $48 \mathrm{~h}$. The results revealed that increasing the concentration doses of cisplatin reduced cell viability in all cell lines (Fig. 2). Tca8113, HN4 and HN30 cells exhibited a higher sensitivity to cisplatin than Cal27 cells. The $\mathrm{IC}_{50}$ values for cisplatin alone at $48 \mathrm{~h}$ in Tca8113, HN30, HN4 and Cal27 cells were 1.685 (1.535-1.835), 2.201 (1.971-2.431), 1.797 (1.722-1.872) and $7.729 \mu \mathrm{g} / \mathrm{ml}(4.162-11.296 \mu \mathrm{g} / \mathrm{ml})$, respectively (Table I). When cisplatin was combined with $5 \mu \mathrm{M} \mathrm{GC7}$, cisplatin sensitivity significantly increased and exerted a stronger antiproliferative effect in all four cell lines compared to cisplatin alone and the $\mathrm{IC}_{50}$ values at $48 \mathrm{~h}$ in Tca8113, HN30, HN4 and Cal27 cells were decreased to $0.818(0.787-0.850, \mathrm{P}<0.05)$, 1.000 (0.955-1.046, $\mathrm{P}<0.05), 1.215(1.164-1.266, \mathrm{P}<0.05)$ and $2.994 \mu \mathrm{g} / \mathrm{ml}$ (2.611-3.37 $\mu \mathrm{g} / \mathrm{ml}, \mathrm{P}<0.05$ ), respectively (Fig. 2 and Table I). Therefore, GC7 significantly sensitized OSCC cells to cisplatin.

The induction of apoptosis in HN30 cells was analyzed by flow cytometry following administration of cisplatin $\left(\mathrm{IC}_{50}\right.$ and $\mathrm{IC}_{30}$ values) alone or in combination with $5 \mu \mathrm{M} \mathrm{GC7}$ for 24 and $48 \mathrm{~h}$, respectively. Notably, co-treatment with GC7 had no evident effect on apoptosis induction compared to cisplatin alone (Fig. 3A, P>0.05).

GC7 sensitizes the cytotoxicity of cisplatin in mesenchymal phenotype OSCC cells through STAT3 pathway inhibition 
A

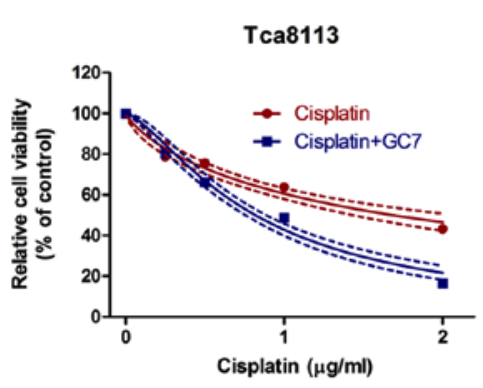

B

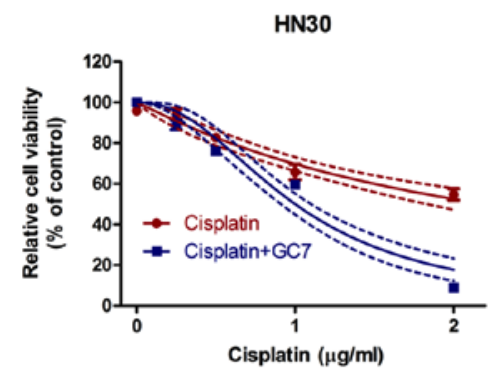

C

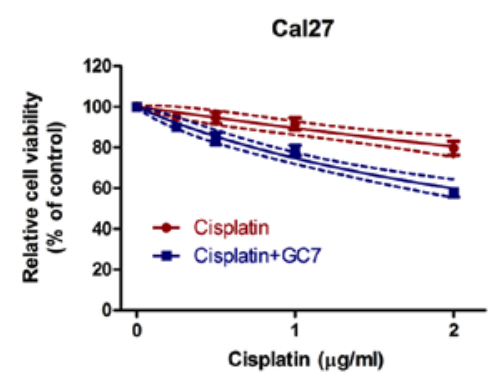

D

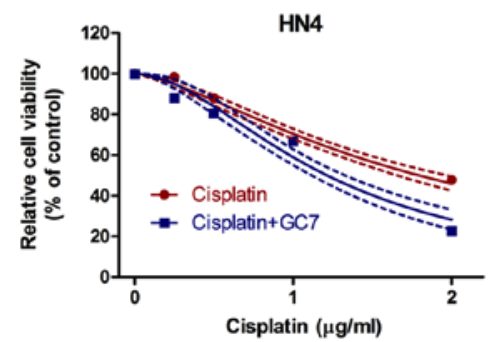

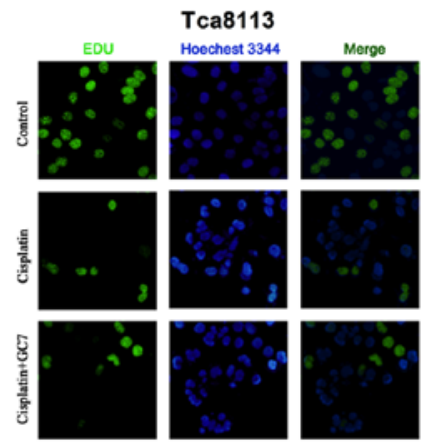

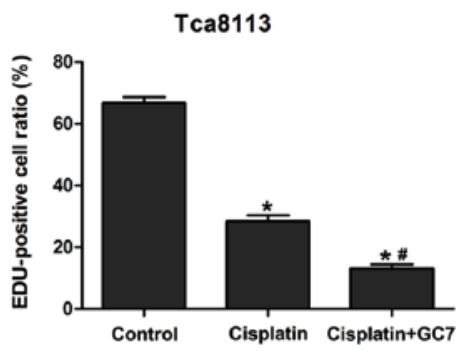

HN30
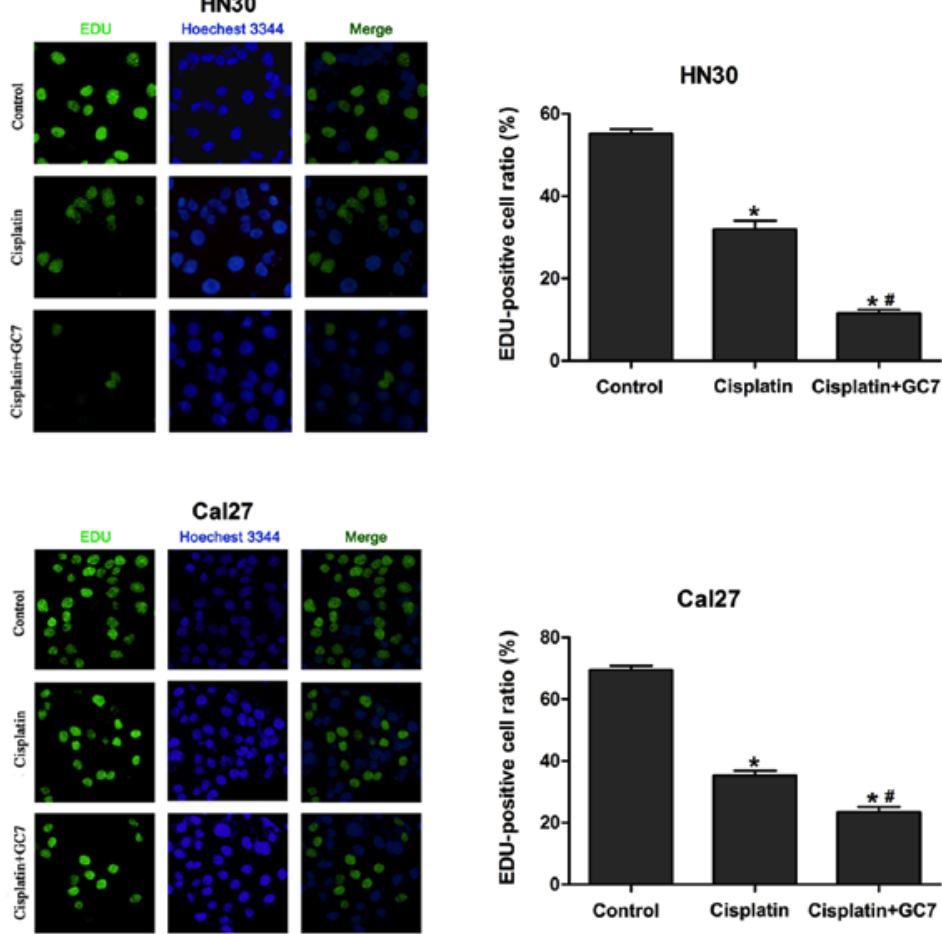

Cal27
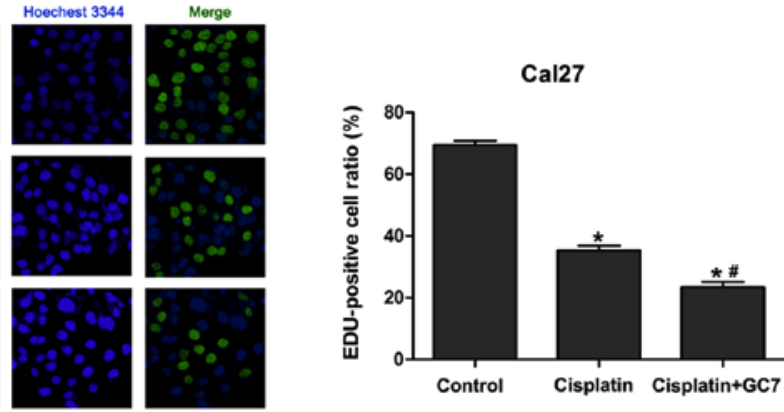

HN4
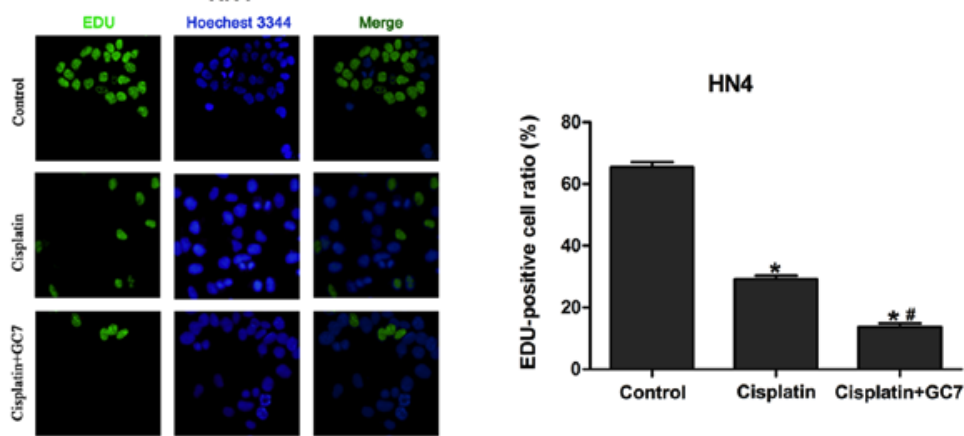

Figure 2. Cytotoxicity of cisplatin is enhanced by low concentrations of GC7 in OSCC cells. GC7 (5 $\mu \mathrm{M})$ significantly enhanced the cytotoxicity of cisplatin in (A) Tca8113, (B) HN30, (C) Cal27 and (D) HN4 cells. The best fit lines (solid) and 95\% confidence intervals (dashed) are indicated for the treatments in different cells. Photomicrographs and bar charts illustrate the 5-ethynyl-2'-deoxyuridine (EdU) staining pattern and relative EdU-positive ratio of different cell lines, after $48 \mathrm{~h}$ of treatment with control, cisplatin alone or combined with GC7 ("P<0.05 for cisplatin vs. control; ${ }^{* \#} \mathrm{P}<0.05$ for cisplatin + GC7 vs. cisplatin). GC7, N1-guanyl-1,7-diaminoheptane; OSCC, oral squamous cell carcinoma

and eIF5A2 inactivation. In order to determine whether the phenotype of OSCC cells contributed to their differing chemosensitivity to combined therapy, we assessed the expression of epithelial/mesenchymal markers in OSCC cells. The results revealed that the E-cadherin/vimentin ratio was clearly higher in the HN4 and Cal27 cells with an epithelial phenotype than in the HN30 and Tca8113 cells, which have a mesenchymal phenotype (Fig. 3B). Notably, mesenchymal OSCC cells exhibited more sensitivity to cisplatin than epithelial OSCC cells. Cisplatin alone reversed EMT in mesenchymal OSCC 
A
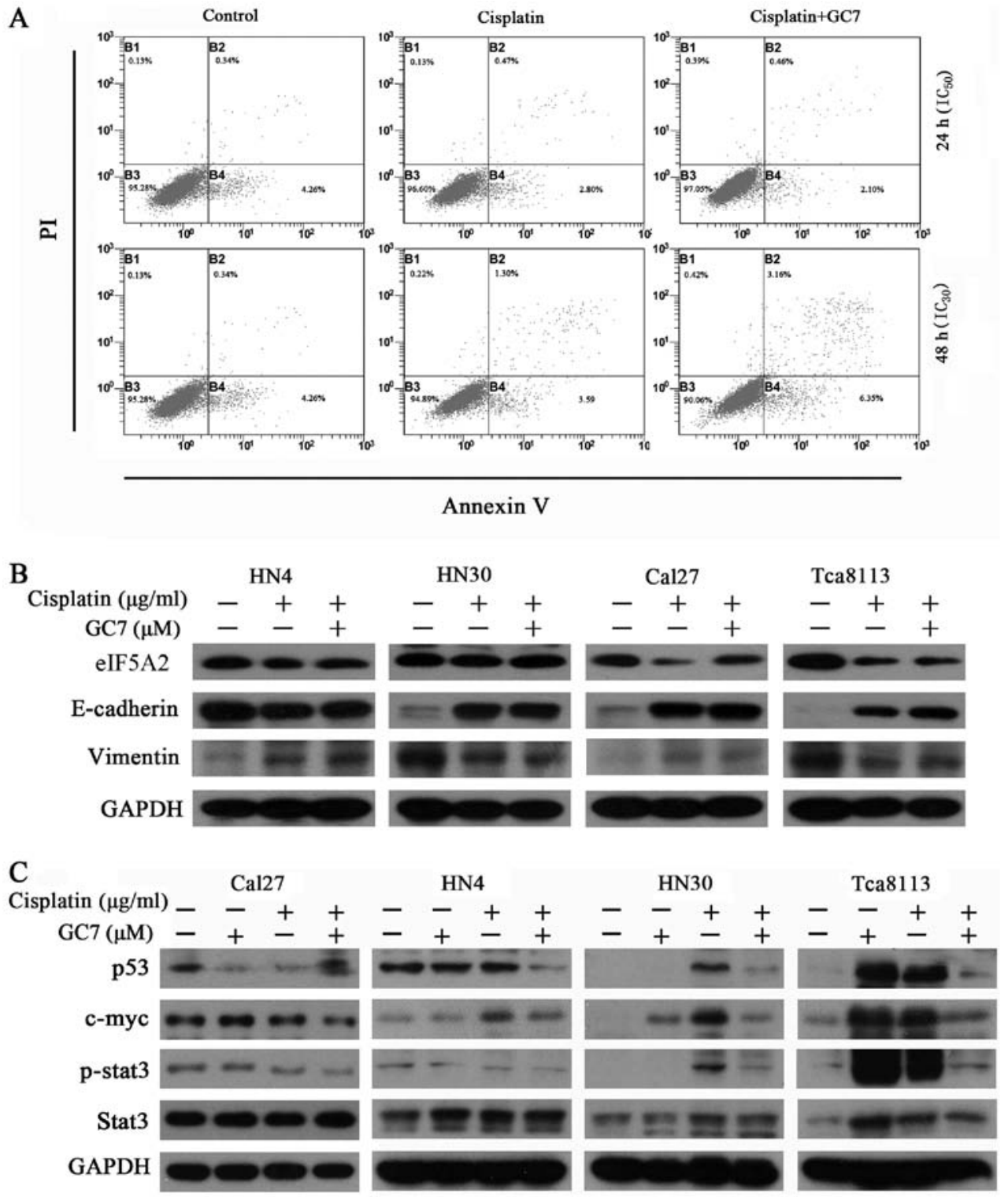

Figure 3. GC7 inhibits cisplatin-induced STAT3 signaling pathway feedback activation in mesenchymal phenotype OSCC cells. (A) Apoptosis flow cytometric analysis images, (B) western blot analysis of the expression of EMT markers (E-cadherin and vimentin) and (C) key components of STAT3 signaling pathway (p-STAT, STAT and c-Myc) in control OSCC cells and OSCC cells treated for $24 \mathrm{~h}$ (for the apoptosis assay) or $48 \mathrm{~h}$ (for the apoptosis assay and western blot analysis) with cisplatin alone or combined with GC7. GC7, N1-guanyl-1,7-diaminoheptane; EMT, epithelial-mesenchymal transition; OSCC, oral squamous cell carcinoma.

cells through upregulation of E-cadherin and downregulation of vimentin. Although cisplatin alone increased the expression of E-cadherin, it also slightly increased the expression of vimentin in epithelial OSCC cells. There were no significant changes in the expression of E-cadherin and vimentin in both epithelial and mesenchymal OSCC cells treated with cisplatin alone as compared to the combined treatment (Fig. 3B). Therefore, the ability of GC7 to enhance the cytotoxicity of cisplatin did not occur in relation to phenotype transition in OSCC cells.

STAT3, one of the seven members of the STAT protein family that regulates cell cycle progression and cell growth, exhibits constitutive activity in various human malignancies, including breast, prostate, head and neck, lung, colon, liver and pancreatic cancers, and lymphoma, leukemia and multiple myeloma (1-3). Notably, we revealed that the ability of GC7 to enhance the cytotoxicity of cisplatin in mesenchymal phenotype OSCC cells may be mediated through STAT3 pathway inhibition and the eIF5A2 inactivation. As shown in Fig. 3B and C, c-Myc and p-STAT3 were upregulated in OSCC cells treated with cisplatin or GC7 alone compared to the control, whereas the combined treatment could significantly reverse this upregulation in mesenchymal phenotype Tca8113 and HN30 cells. Similar results were not observed in epithelial phenotype Cal27 and HN4 cells. Notably, we found that the expression of eIF5A2 was decreased via combined treatment in Tca8113 cells.

To further ascertain the mechanism by which GC7 enhanced the cytotoxicity of cisplatin in OSCC cells, eIF5A2 siRNA was transfected into HN4, Cal27, HN30 and Tca8113 cells to inhibit the eIF5A2 expression and found that the expression of eIF5A2 was significantly inhibited in all four 
A

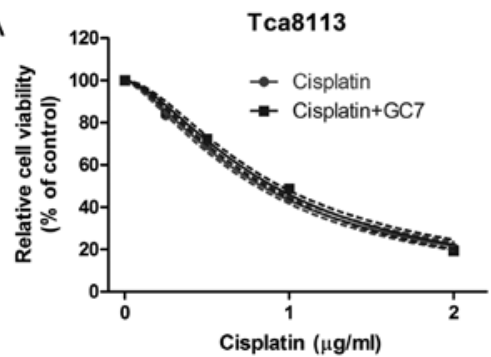

C

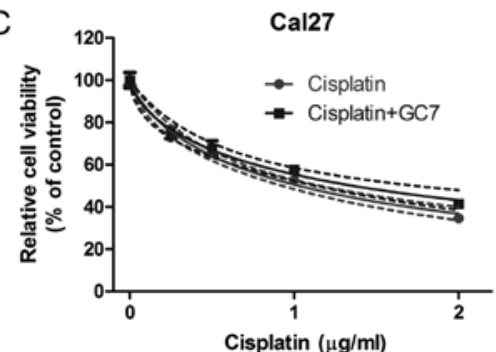

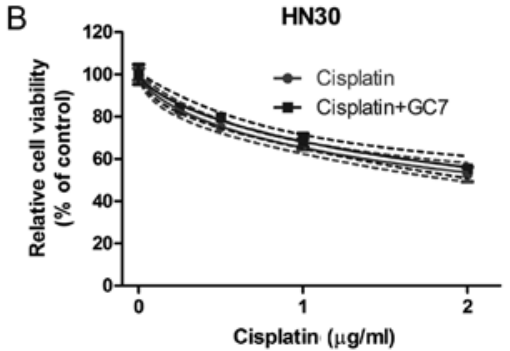

D

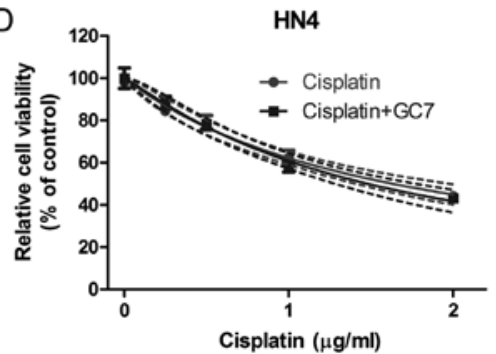

$\mathrm{E}$

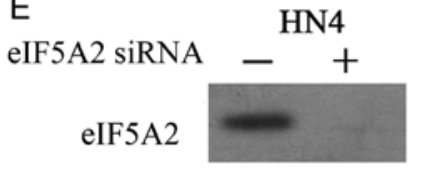

Cal27

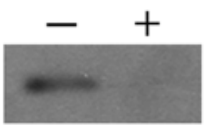

Tca8113

HN30
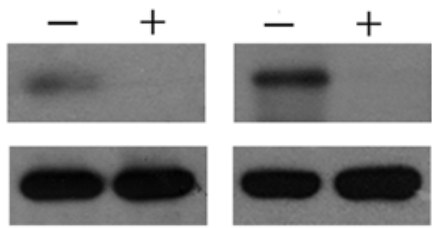

Figure 4. GC7 enhances cisplatin sensitivity in OSCC cells via inhibition of eIF5A2. Knockdown of eIF5A2 by siRNA-transfection reduced the synergistic effect of cisplatin combined with GC7 in (A) Tca8113, (B) HN30, (C) Cal27 and (D) HN4 cells. The best fit lines (solid) and 95\% confidence intervals (dashed) are indicated for the treatments in different cells ( $\mathrm{P}>0.05$ for cisplatin $+\mathrm{GC} 7$ vs. cisplatin). (E) Western blot analysis of expression of eIF5A2 in OSCC cells transfected with eIF5A2 siRNA (+) or negative siRNA (-). GC7, N1-guanyl-1,7-diaminoheptane; OSCC, oral squamous cell carcinoma; eIF5A2, eukaryotic initiation factor $5 \mathrm{~A} 2$.

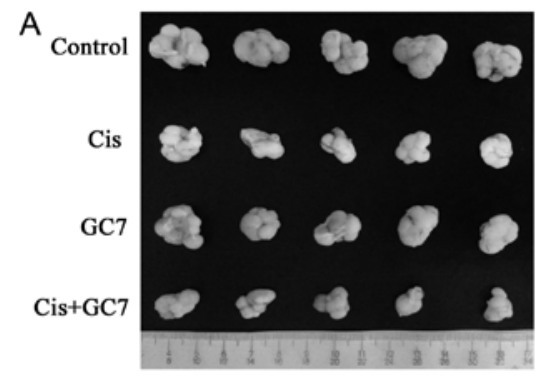

C



B

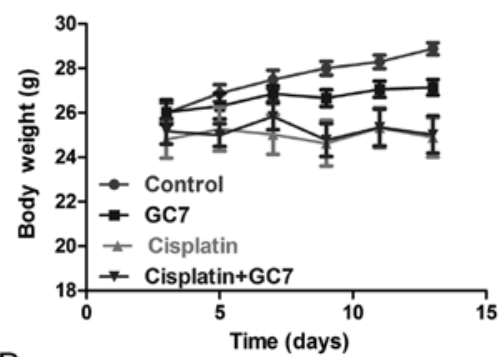

D

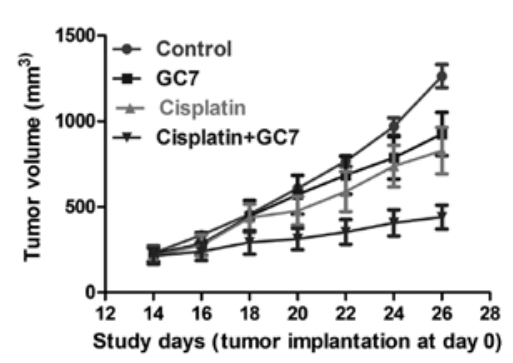

Figure 5. GC7 combined with cisplatin exhibits potent growth inhibition in mesenchymal phenotype OSCC Tca8113 cells in vivo. Tumor volume (D) and body weight (B) from the Tca8113 cell xenograft mice treated with GC7, cisplatin, cisplatin + GC7 or vehicle-treated control groups were assessed every other day as indicated. (A) After 2 weeks of treatment, the mice were euthanized, and tumors were dissected and weighed. (C) The relative tumor inhibitory rates were normalized to the control group $\left({ }^{*} \mathrm{P}<0.05\right.$ for cisplatin vs. GC7; ${ }^{* *} \mathrm{P}<0.05$ for cisplatin + GC7 vs. cisplatin. Dots, mean; bars, SD; GC7, N1-guanyl-1,7diaminoheptane; OSCC, oral squamous cell carcinoma.

OSCC cell lines (Fig. 4E). CCK-8 cell viability assays were then carried out in these transfected OSCC cells treated with cisplatin alone or combined with GC7 for $48 \mathrm{~h}$. The results indicated that increasing the concentration doses of cisplatin 
Table II. $\mathrm{IC}_{50}$ values of cisplatin in eIF5A2-siRNA transfected OSCC cell lines with or without GC7 treatment.

\begin{tabular}{lcc}
\hline & \multicolumn{2}{c}{$\mathrm{IC}_{50}(\mu \mathrm{g} / \mathrm{ml})^{\mathrm{a}}$} \\
\cline { 2 - 3 } OSCC cell lines & Cisplatin & Cisplatin + GC7 \\
\hline Cal27 & $1.030(0.975-1.085)$ & $1.359(1.220-1.498)$ \\
HN4 & $1.637(1.504-1.770)$ & $1.483(1.368-1.598)$ \\
HN30 & $2.452(2.096-2.808)$ & $2.771(2.293-3.249)$ \\
Tca8113 & $0.833(0.816-0.851)$ & $0.901(0.880-0.922)$ \\
\hline
\end{tabular}

${ }^{a} \mathrm{IC}_{50}$ values and $95 \%$ confidence interval of cisplatin in each treatment. OSCC, oral squamous cell carcinoma. $\mathrm{P}<0.05$ vs. cisplatin.

reduced cell viability in all cell lines (Fig. 4A-D). Although the sensitivity to cisplatin alone increased significantly in Cal27 cells after the eIF5A2 siRNA transfection the $\mathrm{IC}_{50}$ value at $48 \mathrm{~h}$ was decreased from $7.729(4.162-11.296)$ to 1.030 $(0.975-1.085 \mu \mathrm{g} / \mathrm{ml})$ (Tables I and II). The Tca8113 cells were the most sensitive to cisplatin alone after eIF5A2 siRNA transfection and the $\mathrm{IC}_{50}$ value at $48 \mathrm{~h}$ was $0.833(0.816-0.851 \mu \mathrm{g} / \mathrm{ml})$ (Table II). However, when cisplatin treatment was combined with GC7 after eIF5A2 siRNA transfection, no significant increase of cisplatin sensitivity was observed in all four OSCC cell lines and the $\mathrm{IC}_{50}$ values at $48 \mathrm{~h}$ in Tca8113, HN30, HN4 and Cal27 cells were 0.901 (0.880-0.922), 2.771 (2.293-3.249), $1.483(1.368-1.598)$ and $1.359 \mu \mathrm{g} / \mathrm{ml}(1.220-1.498 \mu \mathrm{g} / \mathrm{ml})$, respectively (Table II and Fig. 4A-D).

The antitumor effect of cisplatin is boosted by GC7 without evident toxic side effect increase in the Tca8113 cells in vivo. Based on the significant enhancement of cytotoxicity of cisplatin by GC7 in OSCC cells in vitro and the essential role of STAT3 pathway inhibition via eIF5A2 inactivation, we further examined the antitumor effect of the cisplatin/GC7 combination in Tca8113 cells in vivo. As shown in Fig. 5A-C, GC7 alone exhibited a significant antitumor inhibitory rate compared to the control which indicated the crucial role of eIF5A2 activation in OSCC tumorigenesis. Cisplatin alone revealed lower tumor volume. In addition, cisplatin alone resulted into relatively more body weight loss compared to GC7 alone, which may indicate a higher toxic side effect (Fig. 5B and D). The differences of body weight between the cisplatin and cisplatin/GC7 groups were marginal. Furthermore, tumor volume and weight were significantly decreased in the cisplatin/GC7 group as compared to the cisplatin group alone (Fig. 5B and D). Our results strongly indicated that GC7 may enhance the antitumor effect of cisplatin in vivo without causing severe systemic side-effects (body weight change).

\section{Discussion}

The most significant clinical factor responsible for the vast majority of OSCC deaths is not only metastasis to cervical lymph nodes, but also the impact of primary tumors. Despite the progress in surgery and radiation therapy especially in adjuvant or neo-adjuvant chemotherapy, the 5-year survival rate for patients with advanced or recurrent OSCC has not significantly improved over the past 30 years (1). Therefore, an improved, more promising therapeutic intervention to improve the prognosis of OSCC patients is essential. Recently, combined treatment based on chemotherapy drugs or molecularly-targeted agents revealed promising synergistic antitumor effects and relieved the toxic side-effects usually caused by chemotherapy $(4,21,28,29)$. The overexpression of eIF5A2 has been reported to be associated with invasion and metastasis in various human malignances, including colon, bladder, ovarian and hepatocellular cancer $(14,15,17)$. In addition, eIF5A2 has been shown to induce EMT in colorectal and hepatocellular cancer $(22,23,26)$. In the present study, we first demonstrated that GC7, an inhibitor of the eIF5A2 activation, exerted better antitumor effects in the OSCC cells in vitro and in vivo combined with cisplatin-based therapy, the most frequently used chemotherapy drugs in OSCC patients, and then identified the potential molecular mechanism mediating the synergistic antitumor effects.

As the substrate of DHPS, eIF5A2 isolated from 3q26 using chromosome microdissection and hybrid selection, is essential for the proliferation of mammalian cells and has recently been considered as a novel candidate oncogene highly associated to tumor metastasis and invasion (30-33). Despite the fact that the properties of malignancy of various human cancers are closely related with the expression of eIF5A2, the expression and function of eIF5A2 in OSCC is not fully elucidated. In the present study, we observed a high level of eIF5A2 expression in HN4, Cal27, HN30 and Tca8113 cells, while the inactivation of eIF5A2 by GC7 or the knockdown of eIF5A2 expression by siRNA transfection revealed a significant inhibition of proliferation of the OSCC cells in vitro and in vivo. Thus, we came to the conclusion that eIF5A2 may also play a key role in the properties of malignancy of the OSCC cells which may be a potential therapeutic target.

EMT has been demonstrated to be associated with tumorigenicity, growth and invasiveness in various human tumors including OSCC and it has been suggested that EMT may be involved in drug resistance in $\operatorname{OSCC}(7,8,12,34,35)$. In the present study, we found that mesenchymal Tca8113 and HN30 cells were more sensitive to cisplatin compared to epithelial Cal27 and HN4 cells. Compared to the untreated control cells, cisplatin downregulated vimentin and upregulated E-cadherin in the Tca8113 and HN30 cells, which indicated that the cells underwent EMT reversion in response to cisplatin. Furthermore, cisplatin downregulated E-cadherin and upregulated vimentin in the relatively less sensitive Cal27 and HN4 cells which indicated that the cells underwent EMT. However, when treated with the combination of cisplatin and GC7, a novel inhibitor of DHPS which is required for the activation of eIF5A2, the expression of E-cadherin and vimentin did not change, while the sensitivity to cisplatin was significantly inceased compared to cisplatin treatment alone in the OSCC cell lines. This study revealed that GC7 enhanced the cytotoxicity of cisplatin in OSCC cells through the inactivation of eIF5A2 without EMT involvement. To further determine the role of eIF5A 2 in the increased GC7-induced cisplatin sensitivity, the OSCC cells were transfected with eIF5A2 siRNA. Consistent with our 
hypothesis, the synergistic effect of cisplatin and GC7 was eliminated by eIF5A2 siRNA transfection.

STAT3 is one of the seven members of the STAT protein family with SH2 (Src Homology-2) domains that act as signal messengers and transcription factors and mediate the actions of many cytokines and growth factors (11). The constitutive activity of STAT3 has been associated with a wide variety of human malignancies, including breast, prostate, head and neck, lung, colon, liver and pancreatic cancers, and multiple myeloma (36-39). The in vitro data in the present study revealed that the mesenchymal phenotype Tca8113 and HN30 cells were more sensitive to cisplatin compared to the epithelial Cal27 and HN4 cells. To ascertain the molecular mechanisms involved in the combination of GC7 with cisplatin in different phenotype OSCC cells, we observed that cisplatin significantly upregulated p-STAT3 and c-Myc which was abolished when co-treated with GC7 in mesenchymal phenotype Tca8113 and HN30 cells (40). Similar results were not observed in epithelial phenotype Cal27 and HN4 cells, which indicated that GC7 may enhance cisplatin sentivity via other molecular mechanisms in epithelial phenotype Cal27 and HN4 cells. Therefore, our data revealed that the STAT3 signaling pathway may be involved in chemoresistance to cisplatin in more aggressive mesenchymal OSCC cells and that inhibition of eIF5A2 activity could eliminate this effect. The specific mechanisms involved in STAT3 pathway regulation and eIF5A2 activity are worth further investigation in mesenchymal phenotype OSCC cells.

Our aim for the future is not only to increase the efficiency of cisplatin in more aggressive OSCC cells, but more importantly, to reduce the adverse side-effects associated with the administration of high doses of cisplatin. The results obtained with OSCC cells in vitro prompted us to evaluate the efficacy of GC7 in increasing cisplatin sensitivity in vivo. In combination with GC7, it is interesting to note that a significant inhibition of tumor growth was found without causing distinct body weight reduction compared with cisplatin alone. Accordingly, we considered that the potent effect of cisplatin combined with GC7 may be more specific in blocking OSCC cell growth than other normal cells, which could represent a remarkable key point in developing efficient and safe adjuvant treatment of OSCC. Further in vitro and in vivo studies are required to investigate the underlying molecular mechanisms involved in the increase of cisplatin sensitivity via GC7-mediated inactivation of eIF5A2 in OSCC cells, especially with a more aggressive mesenchymal phenotype.

In conclusion, the present study revealed that combined treatment with GC7 enhanced the cytotoxicity of cisplatin in OSCC cells through inhibition of eIF5A2 activation. In addition, this is the first evidence that combined treatment with GC7 presents a significant antineoplastic effect in more aggressive mesenchymal phenotype OSCC cells in vitro and in vivo by preventing cisplatin- or GC7-induced STAT3 signaling pathway activation. Therefore, the combination therapy with GC7 may contribute to a better effect with a lower recurrence rate and adverse side-effects after cisplatin chemotherapy. Collectively, these findings not only provided strong evidence to consider eIF5A as a novel target in OSCC, but also offered a new strategy to improve the treatment of patients with advanced or recurrent OSCC in clinical practice.

\section{Acknowledgements}

The present study was funded by the National Natural Science Foundation of China (grant no. 81302353).

\section{References}

1. Cancer Stat Facts SEER: Oral Cavity and Pharynx Cancer. National Cancer Institute. Bethesda, MD, http://seer.cancer.gov/ statfacts/html/oralcav.html.

2. Rohde S, Kovács AF, Turowski B, Yan B, Zanella F and Berkefeld J: Intra-arterial high-dose chemotherapy with cisplatin as part of a palliative treatment concept in oral cancer. AJNR Am J Neuroradiol 26: 1804-1809, 2005.

3. Kovács AF: Chemoembolization using cisplatin crystals as neoadjuvant treatment of oral cancer. Cancer Biother Radiopharm 20: 267-279, 2005.

4. Andreadis C, Vahtsevanos K, Sidiras T, Thomaidis I, Antoniadis K and Mouratidou D: 5-Fluorouracil and cisplatin in the treatment of advanced oral cancer. Oral Oncol 39: 380-385, 2003.

5. da Silva SD, Hier M, Mlynarek A, Kowalski LP and AlaouiJamali MA: Recurrent oral cancer: Current and emerging therapeutic approaches. Front Pharmacol 3: 149, 2012.

6. Mehrotra R, Ibrahim R, Eckardt A, Driemel O and Singh M: Novel strategies in therapy of head and neck cancer. Curr Cancer Drug Targets 11: 465-478, 2011.

7. Smith A, Teknos TN and Pan Q: Epithelial to mesenchymal transition in head and neck squamous cell carcinoma. Oral Oncol 49: 287-292, 2013.

8. Chang JY, Wright JM and Svoboda KK: Signal transduction pathways involved in epithelial-mesenchymal transition in oral cancer compared with other cancers. Cells Tissues Organs 185: 40-47, 2007.

9. Kajiyama H, Shibata K, Terauchi M, Yamashita M, Ino K, Nawa A and Kikkawa F: Chemoresistance to paclitaxel induces epithelial-mesenchymal transition and enhances metastatic potential for epithelial ovarian carcinoma cells. Int J Oncol 31: 277-283, 2007.

10. Lewin B, Siu A, Baker C, Dang D, Schnitt R, Eisapooran P and Ramos DM: Expression of Fyn kinase modulates EMT in oral cancer cells. Anticancer Res 30: 2591-2596, 2010.

11. Sasahira T, Kirita T and Kuniyasu H: Update of molecular pathobiology in oral cancer: A review. Int J Clin Oncol 19: 431-436, 2014.

12. Attramadal CG, Kumar S, Boysen ME, Dhakal HP, Nesland JM and Bryne M: Tumor budding, EMT and cancer stem cells in T1-2/N0 oral squamous cell carcinomas. Anticancer Res 35: 6111-6120, 2015.

13. Zhang Z, Filho MS and Nör JE: The biology of head and neck cancer stem cells. Oral Oncol 48: 1-9, 2012.

14. Mathews MB and Hershey JWB: The translation factor eIF5A and human cancer. Biochim Biophys Acta 1849: 836-844, 2015.

15. Clement PMJ, Henderson CA, Jenkins ZA, Smit-McBride Z, Wolff EC, Hershey JW, Park MH and Johansson HE: Identification and characterization of eukaryotic initiation factor 5A-2. Eur J Biochem 270: 4254-4263, 2003.

16. Xing ZH, Wei JH, Cheang TY, Wang ZR, Zhou X, Wang SS, Chen W, Wang SM, Luo JH and Xu AW: Bifunctional pH-sensitive $\mathrm{Zn}(\mathrm{II})$-curcumin nanoparticles/siRNA effectively inhibit growth of human bladder cancer cells in vitro and in vivo. J Mater Chem B Mater Biol Med 2: 2714-2724, 2014.

17. Wang FW, Guan XY and Xie D: Roles of eukaryotic initiation factor 5A2 in human cancer. Int J Biol Sci 9: 1013-1020, 2013.

18. Tang DJ, Dong SS, Ma NF, Xie D, Chen L, Fu L, Lau SH, Li Y, Li Y and Guan XY: Overexpression of eukaryotic initiation factor 5A2 enhances cell motility and promotes tumor metastasis in hepatocellular carcinoma. Hepatology 51: 1255-1263, 2010.

19. Liu Y, Liu R, Fu P, Du F, Hong Y, Yao M, Zhang X and Zheng S: N1-Guanyl-1,7-diaminoheptane sensitizes estrogen receptor negative breast cancer cells to doxorubicin by preventing epithelial-mesenchymal transition through inhibition of eukaryotic translation initiation factor 5A2 activation. Cell Physiol Biochem 36: 2494-2503, 2015. 
20. Yang J, Yu H, Shen M, Wei W, Xia L and Zhao P: N1-guanyl-1,7diaminoheptane sensitizes bladder cancer cells to doxorubicin by preventing epithelial-mesenchymal transition through inhibition of eukaryotic translation initiation factor 5A2 activation. Cancer Sci 105: 219-227, 2014.

21. Xu G, Yu H, Shi X, Sun L, Zhou Q, Zheng D, Shi H, Li N, Zhang X and Shao G: Cisplatin sensitivity is enhanced in non-small cell lung cancer cells by regulating epithelial-mesenchymal transition through inhibition of eukaryotic translation initiation factor 5A2. BMC Pulm Med 14: 174, 2014

22. Lou B, Fan J, Wang K, Chen W, Zhou X, Zhang J, Lin S, Lv F and Chen Y: N1-guanyl-1,7-diaminoheptane (GC7) enhances the therapeutic efficacy of doxorubicin by inhibiting activation of eukaryotic translation initiation factor 5A2 (eIF5A2) and preventing the epithelial-mesenchymal transition in hepatocellular carcinoma cells. Exp Cell Res 319: 2708-2717, 2013.

23. Bao Y, Lu Y, Wang X, Feng W, Sun X, Guo H, Tang C, Zhang X, Shi Q and $\mathrm{Yu} \mathrm{H}$ : Eukaryotic translation initiation factor 5A2 (eIF5A2) regulates chemoresistance in colorectal cancer through epithelial mesenchymal transition. Cancer Cell Int 15: 109, 2015.

24. Tomayko MM and Reynolds CP: Determination of subcutaneous tumor size in athymic (nude) mice. Cancer Chemother Pharmacol 24: $148-154,1989$

25. Dykes DJ, Harrison SD Jr, Mayo JG and Griswold DP Jr: Excision assay for initial evaluation of antitumor drug activity in mice bearing human tumor xenografts. J Natl Cancer Inst 84 528-530, 1992

26. Lee NP, Tsang FH, Shek FH, Mao M, Dai H, Zhang C, Dong S, Guan XY, Poon RT and Luk JM: Prognostic significance and therapeutic potential of eukaryotic translation initiation factor $5 \mathrm{~A}$ (eIF5A) in hepatocellular carcinoma. Int J Cancer 127: 968-976, 2010.

27. Nakanishi S and Cleveland JL: Targeting the polyamine-hypusine circuit for the prevention and treatment of cancer. Amino Acids 48: 2353-2362, 2016.

28. Wang L, Mosel AJ, Oakley GG and Peng A: Deficient DNA damage signaling leads to chemoresistance to cisplatin in oral cancer. Mol Cancer Ther 11: 2401-2409, 2012.

29. Yu ZW, Zhong LP, Ji T, Zhang P, Chen WT and Zhang CP: MicroRNAs contribute to the chemoresistance of cisplatin in tongue squamous cell carcinoma lines. Oral Oncol 46: 317-322, 2010
30. Chen Z, Yu T, Zhou B, Wei J, Fang Y, Lu J, Guo L, Chen W, Liu ZP and Luo J: Mg(II)-Catechin nanoparticles delivering siRNA targeting EIF5A2 inhibit bladder cancer cell growth in vitro and in vivo. Biomaterials 81: 125-134, 2016.

31. Tian SB, Yu JC, Liu YQ, Kang WM, Ma ZQ, Ye X and Yan C: MiR-30b suppresses tumor migration and invasion by targeting EIF5A2 in gastric cancer. World J Gastroenterol 21: 9337-9347, 2015.

32. Meng QB, Kang WM, Yu JC, Liu YQ, Ma ZQ, Zhou L, Cui QC and Zhou WX: Overexpression of eukaryotic translation initiation factor 5A2 (EIF5A2) correlates with cell aggressiveness and poor survival in gastric cancer. PLoS One 10: e0119229, 2015.

33. Liu Y, Du F, Chen W, Yao M, Lv K and Fu P: EIF5A2 is a novel chemoresistance gene in breast cancer. Breast Cancer 22: 602-607, 2015.

34. Lee WY, Shin DY, Kim HJ, Ko YH, Kim S and Jeong HS: Prognostic significance of epithelial-mesenchymal transition of extracapsular spread tumors in lymph node metastases of head and neck cancer. Ann Surg Oncol 21: 1904-1911, 2014.

35. Harada K, Ferdous T and Ueyama Y: Establishment of 5-fluorouracil-resistant oral squamous cell carcinoma cell lines with epithelial to mesenchymal transition changes. Int J Oncol 44: 1302-1308, 2014

36. Aggarwal BB, Sethi G, Ahn KS, Sandur SK, Pandey MK, Kunnumakkara AB, Sung B and Ichikawa H: Targeting signaltransducer-and-activator-of-transcription-3 for prevention and therapy of cancer: Modern target but ancient solution. Ann NY Acad Sci 1091: 151-169, 2006.

37. Yadav A, Kumar B, Datta J, Teknos TN and Kumar P: IL-6 promotes head and neck tumor metastasis by inducing epithelialmesenchymal transition via the JAK-STAT3-SNAIL signaling pathway. Mol Cancer Res 9: 1658-1667, 2011.

38. Gkouveris I, Nikitakis N, Karanikou M, Rassidakis G and Sklavounou A: Erk1/2 activation and modulation of STAT3 signaling in oral cancer. Oncol Rep 32: 2175-2182, 2014.

39. Buettner R, Mora LB and Jove R: Activated STAT signaling in human tumors provides novel molecular targets for therapeutic intervention. Clin Cancer Res 8: 945-954, 2002.

40. Zhu W, Cai MY, Tong ZT, Dong SS, Mai SJ, Liao YJ, Bian XW, Lin MC, Kung HF, Zeng YX, et al: Overexpression of EIF5A2 promotes colorectal carcinoma cell aggressiveness by upregulating MTA1 through C-myc to induce epithelialmesenchymaltransition. Gut 61: 562-575, 2012. 